\title{
On-demand pulsatile intracerebral delivery of carisbamate with closed-loop direct neurostimulation therapy in an electrically induced self-sustained focal-onset epilepsy rat model
}

\author{
Erwin Z. Mangubat, MD, MPH, ${ }^{1}$ Robert G. Kellogg, MD, ${ }^{1}$ Timothy J. Harris Jr., ${ }^{2}$ \\ and Marvin A. Rossi, MD, PhD² \\ Departments of ${ }^{1}$ Neurosurgery and ${ }^{2}$ Neurological Sciences, Rush University Medical Center, Chicago, Illinois
}

OBJECT The authors evaluated the preclinical feasibility of acutely stabilizing an active bihemispheric limbic epileptic circuit using closed-loop direct neurostimulation therapy in tandem with "on-demand"' convection-enhanced intracerebral delivery of the antiepileptic drug (AED) carisbamate. A rat model of electrically induced self-sustained focal-onset epilepsy was employed.

METHODS A 16-contact depth-recording microelectrode was implanted bilaterally in the dentate gyrus (DG) of the hippocampus of Fischer 344 rats. The right microelectrode array included an integrated microcatheter for drug delivery at the distal tip. Bihemispheric spontaneous self-sustained limbic status epilepticus (SSLSE) was induced in freely moving rats using a 90-minute stimulation paradigm delivered to the right medial perforant white matter pathway. Immediately following SSLSE induction, closed-loop right PP stimulation therapy concurrent with on-demand nanoboluses of the AED $\left[{ }^{14} \mathrm{C}\right]$-carisbamate $(n=4)$, or on-demand $\left[{ }^{14} \mathrm{C}\right]$-carisbamate alone $(n=4)$, was introduced for a mean of 10 hours. In addition, 2 reference groups received either closed-loop stimulation therapy alone $(n=4)$ or stimulation therapy with saline vehicle only $(n=4)$. All animals were sacrificed after completing the specified therapy regimen. In situ $\left[{ }^{14} \mathrm{C}\right]$-autoradiography was used to determine AED distribution.

RESULTS Closed-loop direct stimulation therapy delivered unilaterally in the right PP aborted ictal runs detected in either ipsi- or contralateral hippocampi. Freely moving rats receiving closed-loop direct stimulation therapy with ondemand intracerebral carisbamate delivery experienced a significant reduction in seizure frequency $(p<0.001)$ and minimized seizure frequency variability during the final $50 \%$ of the therapy/recording session compared with closed-loop stimulation therapy alone.

CONCLUSIONS Unilateral closed-loop direct stimulation therapy delivered to afferent hippocampal white matter pathways concurrent with on-demand ipsilateral intracerebral delivery of nano-bolused carisbamate can rapidly decrease the frequency of electrographic seizures in an active bihemispheric epileptic network. Additionally, direct pulsatile delivery of carisbamate can stabilize seizure frequency variability compared with direct stimulation therapy alone.

http://thejns.org/doi/abs/10.3171/2015.1.JNS14946

KEY WORDS deep brain stimulation; convection enhanced drug delivery; microfluidic electrode; white matter; NeuroPace; epilepsy; responsive neurostimulation; neuromodulation

$\mathrm{D}$ ESPITE treatment with antiepileptic medications, approximately $30 \%$ of patients with epilepsy continue to experience seizures. ${ }^{5}$ Pharmacological resistance in focal-onset epilepsy is defined as the failure of at least 2 appropriate antiepileptic drugs (AEDs) at maximally tolerated doses, with a low probability of becoming seizure free with additional AEDs. ${ }^{13}$ A localizable epilep- togenic source or focus is present within a subset of these patients, and this group is likely to respond to time-tested resective surgery. Unfortunately, the remaining pharmacoresistant patient population will not meet criteria for resective surgery. Such patients possess one or more epileptogenic sources involving eloquent cortex or have elusive foci or epileptic networks.

ABBREVIATIONS AD = afterdischarge; $A E D=$ antiepileptic drug; BBB= blood-brain barrier; $C E D=$ convection-enhanced delivery; $C H S=$ continuous hippocampal stimulation; DG = dentate gyrus; ECOG = electrocorticography; PP = perforant pathway; SSLSE = self-sustained limbic status epilepticus; TTL = transistor-transistor logic.

SUBMITTED May 12, 2014. ACCEPTED January 5, 2015.

INCLUDE WHEN CITING Published online February 27, 2015; DOI: 10.3171/2015.1.JNS14946.

DISCLOSURE This study was supported by an investigator-initiated research grant from Ortho-McNeil Janssen Scientific Affairs, LLC. 
The underlying mechanisms of pharmaco-resistant epilepsy are multifactorial. ${ }^{18,34}$ A major factor that limits systemic administration of AEDs is the unwanted side effects of the therapeutic agent on brain systems not involved in the epileptogenic process. In focal-onset epilepsies, side effects and systemic toxicities can be minimized or avoided by restricting delivery of the therapeutic agent to a limited region of brain. ${ }^{27}$ Convection-enhanced delivery (CED) has shown some promise for treating certain neurological diseases by circumventing the blood-brain barrier (BBB) and, therefore, bypassing BBB-associated AED transporters that can contribute to pharmacoresistance. ${ }^{34}$ CED's ability to deliver therapeutics directly to the central nervous system parenchyma provides a potential approach for more effectively treating refractory focal-onset epilepsies., ${ }^{3,8,26}$

In recent years, implantable direct brain stimulation therapy has gained momentum for the treatment of pharmaco-resistant epilepsy. The Responsive Neurostimulation System (RNS, NeuroPace, Inc.) is a closed-loop implantable direct neuromodulation technology recently approved by the FDA as an adjunctive therapy in individuals who have pharmaco-resistant focal-onset epilepsy with up to 2 epileptogenic sources. Efficacy of the NeuroPace RNS capitalizes on a closed-loop approach where direct stimulation is delivered to an epileptogenic source upon detection of an evolving electrographic seizure with the goal of inhibiting its development and propagation. A recent pivotal, randomized controlled trial evaluating the efficacy of the NeuroPace RNS has demonstrated a statistically significant reduction in seizures in the stimulation arm compared with the sham phase..$^{25}$ As a result, the FDA Neurological Devices Panel unanimously recommended the approval of the NeuroPace RNS in February 2103. The system received final FDA approval in late 2013.

This study examines the feasibility of augmenting termination of the seizure onset in a rat model of focal-onset epilepsy through a combination of responsive direct brain stimulation and on-demand nano-volume bolused intracerebral CED of a novel AED, carisbamate (RWJ 333369, Ortho-McNeil Janssen; now known as YKP509, SK Biopharmaceuticals). Carisbamate has previously been proven to be an effective AED in animal models of focal-onset epilepsy and infantile spasms. ${ }^{14,29,38}$ Unlike established AEDs (carbamazepine, phenytoin, lamotrigine, and topiramate), carisbamate demonstrates activity in the lamotrigine-resistant amygdala kindled rat. ${ }^{14}$ Carisbamate's efficacy against recurrent spontaneous seizures in the kainate poststatus epilepticus model $^{7}$ and the GAERS model of spike-wave epilepsy has been previously proven. ${ }^{2}$ This study uses the electrically induced self-sustained limbic status epilepticus (SSLSE) epilepsy model in rats modified from Lothman et al. ${ }^{19}$ and Mazarati et al. ${ }^{22}$

\section{Methods}

\section{Animals}

Twenty-eight Fischer 344 male rats (weight 275-355 g) were obtained from the Harlan Sprague-Dawley Laboratories. The experiments were performed in accordance with the policies of the National Institutes of Health and the Rush University Institutional Animal Care and Use Committee. All animals were individually housed and acclimated for at least 10 days upon arrival from the Harlan colony. They were kept under environmentally controlled conditions (12-hour light/dark cycle at $20^{\circ} \mathrm{C}-23^{\circ} \mathrm{C}$ ) and $50 \%$ relative humidity with ad libitum Wayne food pellets and water.

\section{Surgery}

Preoperatively, each animal was anesthetized with intraperitoneal pentobarbital $(50 \mathrm{mg} / \mathrm{kg})$. Hair was removed from the rat's head with an electric shaver. The skin was prepared with a $1 \%$ tincture of iodine and chlorhexidinebased scrub product. A customized 16-contact microelectrode probe with an integrated 0.25 -mm-inner-diameter stainless steel catheter (NeuroNexus Technologies) (Fig. 1) was implanted in the right hippocampal dentate gyrus (DG) $(1.4 \mathrm{~mm}$ lateral to bregma, depth $4.2 \mathrm{~mm}$ from cortical surface). ${ }^{30}$ A 16-contact microelectrode without fluidic capabilities (NeuroNexus Technologies) was implanted in the left DG. Due to limited recording instrumentation capabilities, only 8 contacts were used on each side. A twisted pair of Teflon-coated stainless steel bipolar stimulating electrode wires was positioned in the right middle perforant pathway (PP) $(8 \mathrm{~mm}$ posterior and $2 \mathrm{~mm}$ lateral to bregma). The PP in the rat demonstrates well-developed axonal afferents to the hippocampal formation. ${ }^{1}$ These inputs are particularly robust in the DG. The stimulating electrode was stereotactically lowered to a depth that, upon stimulation, would result in a characteristic evoked potential from each contact in the dentate gyri bilaterally. Additionally, final implant depth coordinates of the DG microelectrodes were guided by evoking neuroanatomically specific electrophysiological signatures via the right PP stimulating electrode. Specifically, evoked waveforms consisting of an excitatory postsynaptic potential and su-

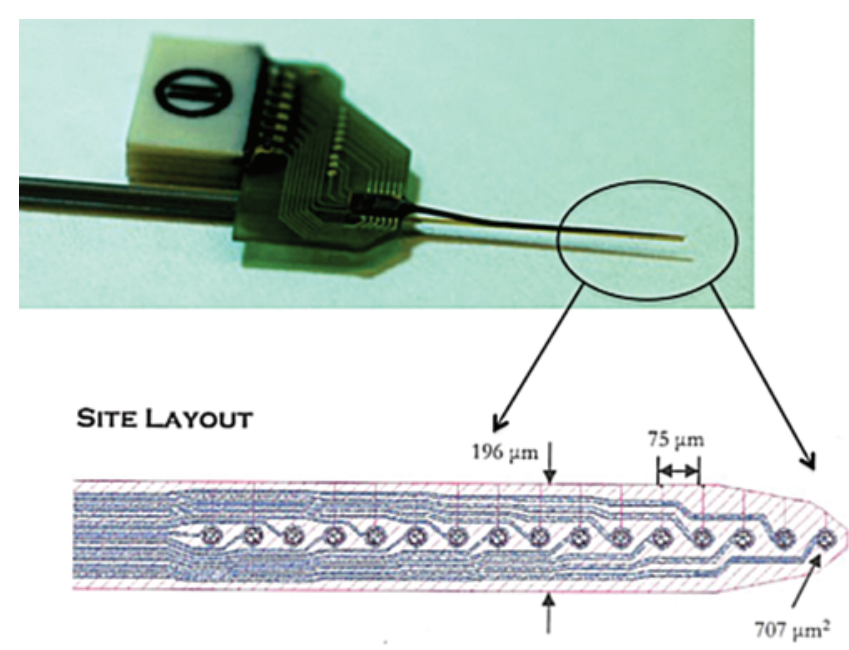

FIG. 1. Photograph of the drug delivery, stimulation, and recording device used in this study (upper) and schematic diagram of the electrode array showing the position of the 16 stimulating/recording microcontacts that were lithographed onto the lead (lower). The microcatheter was used to release $\left[{ }^{14} \mathrm{C}\right]$-carisbamate in Groups 2 and 3 and saline in Group 4. Figure is available in color online only. 
perimposed population spike characteristic of the middle molecular layer of the DG guided placement of the dorsal hippocampal recording microelectrodes bilaterally (Fig. 2). The stimulating electrode positioned in the right PP was used both to induce the SSLSE model and subsequently to deliver closed-loop stimulation therapy throughout the bilateral hippocampal epileptic circuit. A ground screw was placed in the left midcranium and then connected to a ground wire from each microelectrode probe. Two anchor screws were set via a bur hole in the right frontal and left parietal regions of the skull. Cerebond acrylic (Plastics One, Inc.) was then used to fix all electrodes in place.

\section{SSLSE Induction}

For 6 days after electrode implantation, animals received flunixin meglumine $(1.5 \mathrm{mg} / \mathrm{kg}$ administered subcutaneously) and buprenorphine $(0.05-\mathrm{mg} / \mathrm{kg}$ administered subcutaneously) administered every 12 hours for pain control. Immediately after this postsurgical recovery period, a protocol for inducing in vivo SSLSE in the hippocampal network was used as a robust model of producing spontaneous recurring limbic seizures. ${ }^{1,20,35}$ Specifically, afterdischarge (AD) thresholds (measured in microamperes) were determined immediately following the 6-day recov- ery period. AD thresholds were recorded with a standard stimulus of a 10-second train (biphasic 50-Hz, 1-msec pulse width square waves). The AD threshold used for the stimulation protocol was related to the severity of the stimulation-induced behavioral seizures scored according to the modified Racine scale. ${ }^{31}$ The modified Racine scale uses the following designations: Stage 0, no apparent change in behavior; Stage 1, behavioral arrest with mouth/ facial movements; Stage 2, head nodding; Stage 3, forelimb clonus; Stage 4, rearing; and Stage 5, rearing and loss of balance. To study robust, spontaneous seizures, the current intensity chosen was the one that would elicit Stage 3 and 4 behavioral seizures.

The continuous hippocampal stimulation (CHS) protocol was initiated immediately following the determination of AD thresholds. The animals were unanesthetized and freely moving during CHS. CHS consisted of continuous electrical stimulation delivered to the hippocampal formation $(50-\mathrm{Hz}, 1-\mathrm{msec}$ pulse width, 400- $\mu \mathrm{A}$ peak-to-peak square waves). A program was executed within Experimenter Workbench (DataWave Technologies) to trigger an S8800 dual-channel stimulator (Grass Technologies) connected to a Grass current-isolation unit. This setup delivered 9 consecutive 10-minute stimulus epochs. For

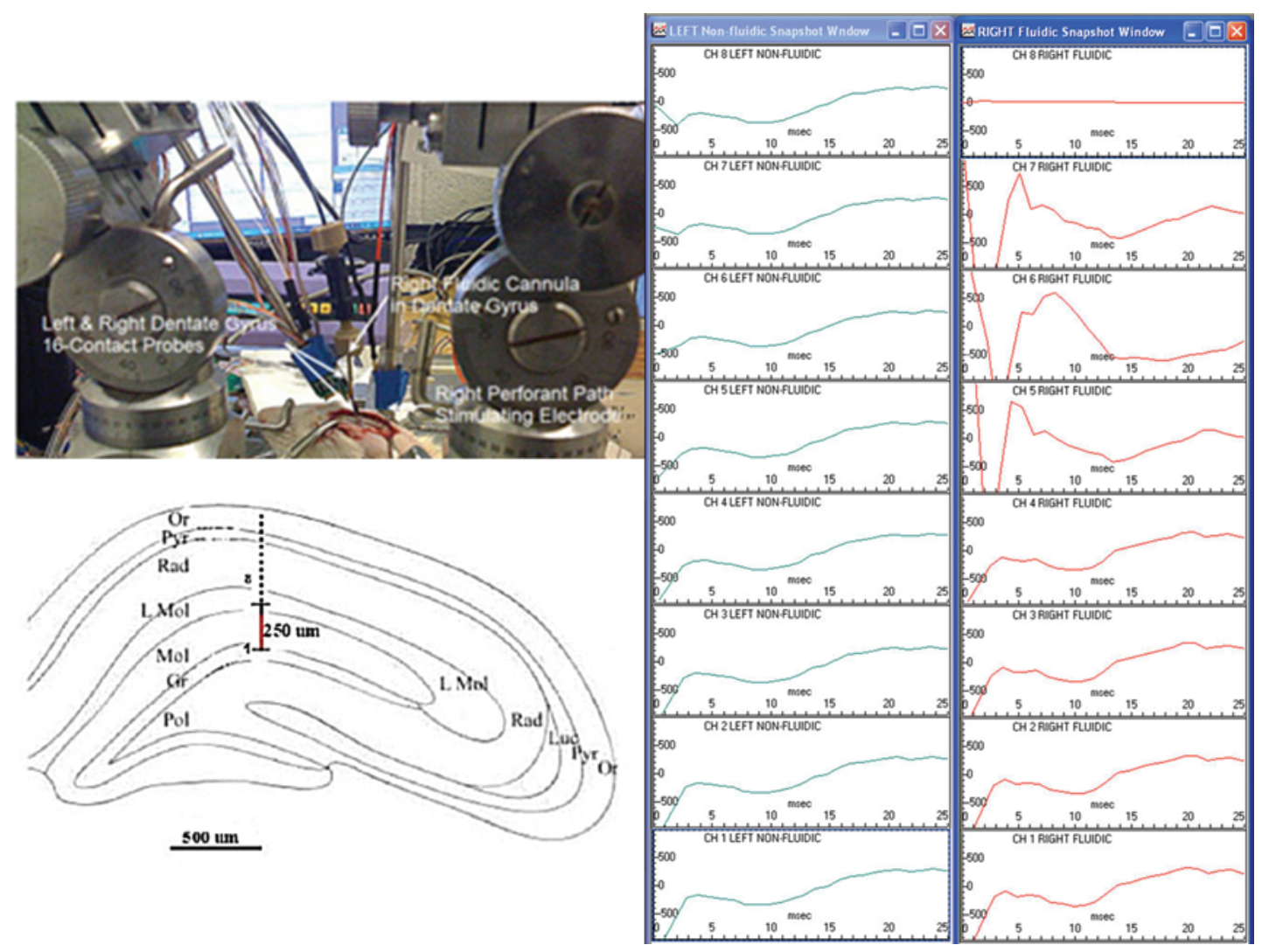

FIG. 2. The distal contacts 1-8 of the left (non-fluidic) and right (fluidic) hemispheric microelectrode probe shafts targeted the middle molecular layer ( $\mathrm{Mol}$ ) of the dentate gyrus (DG) bilaterally. Microelectrode placement was guided and confirmed using extracellular evoked potentials (right channels 5-7). Stimuli were delivered through the right perforant pathway (PP) twisted bipolar electrode. $\mathrm{CH}=$ channel; $\mathrm{Gr}=$ stratum granulosum; $\mathrm{L}$ Mol = lacunosum molecular layer; $\mathrm{Luc}=$ stratum lucidum; Mol =stratum moleculare; Or = stratum oriens; $\mathrm{Pol}=$ polymorphic layer; $\mathrm{Pyr}=$ pyramidal cell layer; $\mathrm{Rad}=$ stratum radiatum. Figure is available in color online only. 
9 minutes of each epoch, stimulation was delivered in the following pattern: 10 seconds "on" and 1 second "off." During the 10th minute, the stimulation was stopped to allow a stimulus-free observation period. Overall, the total duration of CHS was 90 minutes.

\section{Seizure Detection and Therapy}

Extracellularly recorded brain activity was acquired using a cable connected directly to a data processing and display system (DataWave Technologies). Seizure detection was based on a custom-programmed line-length signal-processing tool used to compare paroxysmal electrocorticography (ECOG) waveform patterns over a 10 -second interval versus 2-minute baseline interictal trending. Detection of electrocerebral seizure patterns occurred when a short-term ECOG trend exceeded the long-term trend by a programmed threshold difference. Line-length threshold parameters were established between $10 \%$ and $30 \%$ of baseline ECOG for each animal during the recording of a 2-hour baseline epoch immediately following SSLSE induction and prior to introduction of a therapy regimen. During the therapy testing session (immediately following the post-SSLSE baseline epoch) the same animal-specific line-length thresholds for triggering therapy were used throughout the 10 -hour therapy session. Automated transistor-transistor logic (TTL)-triggered directstimulation therapy was always delivered to the right PP electrode with the aim of influencing the hippocampal circuit bilaterally (see regimen conditions in Fig. 3).

Stimulation therapy parameters were systematically determined as follows. Animal-specific therapeutic stimulus intensities were limited by the available settings of the current-feedback amplifier. In general, the therapeutic stimulus intensities were set at $100 \mu \mathrm{A}$ below AD threshold; however, $500 \mu \mathrm{A}$ was set for therapy when the AD threshold was $750 \mu \mathrm{A}$. In addition, a stimulus frequency of $50 \mathrm{~Hz}$, pulse duration of $1 \mathrm{msec}$, and burst duration of $100 \mathrm{msec}$ were employed to impact $\mathrm{AD}$ frequency and/or duration.

An automated TTL-pulse trigger was used to administer nano-liter boluses of $\left[{ }^{14} \mathrm{C}\right]$-radiolabeled carisbamate (provided by Ortho-McNeil Janssen Scientific Affairs, LLC) utilizing a nano-liter syringe delivery pump (Harvard Apparatus). A 50- $\mu$ l Hamilton syringe was loaded into the pump and connected with 1/32-inch HPLC (high performance liquid chromatography) tubing. The tubing was passed through the cage roof and tethered to the hybrid fluidic catheter using UpChurch connectors (UpChurch Scientific). Regimen conditions included delivery of direct neurostimulation therapy either in the presence or absence of concurrently triggered release of $20-\mathrm{nl}\left[{ }^{14} \mathrm{C}\right]-$ carisbamate boluses into the right DG.

\section{Experimental Protocol}

Following induction of SSLSE, the animal was placed randomly in 1 of 4 groups for receiving closed-loop focal stimulation therapy delivered to the right PP. Stimulation therapy was delivered over 8 hours either in the absence (Group 1, stimulation only; Group 4, stimulation + vehicle) or presence (Group 2, carisbamate in vehicle, without stimulation; Group 3, stimulation + carisbamate and vehicle) of on-demand 20 -nl boluses of $\left[{ }^{14} \mathrm{C}\right]$-carisbamate at a rate of $8.33 \mathrm{nl} / \mathrm{second}$. The drug was always delivered into the right DG. Spontaneous electrographic seizure frequency and duration were assessed over 30-minute intervals during a 10 -hour recording/therapy session (28,800 seconds) following a 2-hour (7200-second) post-SSLSE/pretherapy baseline interval. In many instances, the pump was retriggered during the drug delivery phase, which interrupted delivery of the full 20-nl volume. In these instances only a partial treatment dose was delivered. Therefore, quantifying a total dose delivery of carisbamate during each therapy session was not possible. A blinded evaluator (M.A.R.) reviewed the entirety of each video ECOG session and tabulated the acquired electrographic epileptiform data.

\section{Histology}

Sacrifice of the rats and processing of their brain tissue occurred immediately after the 8- to 10 -hour recording session. All rats were deeply anesthetized with pentobarbital $(100 \mathrm{mg} / \mathrm{kg}$, administered intraperitoneally) prior to sacrifice by decapitation. Immediately prior to decapitation, animals in Group 1 (stimulation therapy only) received a $10-\mu 1$ injection via a Hamilton syringe of fluorescein-labeled chitosan nanoparticles (200-nm diameter) in phosphate-buffered saline (Phosphorex, Inc.) through the right hippocampal microcatheter. Each brain was dissected free from the skull in approximately 120 seconds and then submerged

\section{Regimen Conditions}

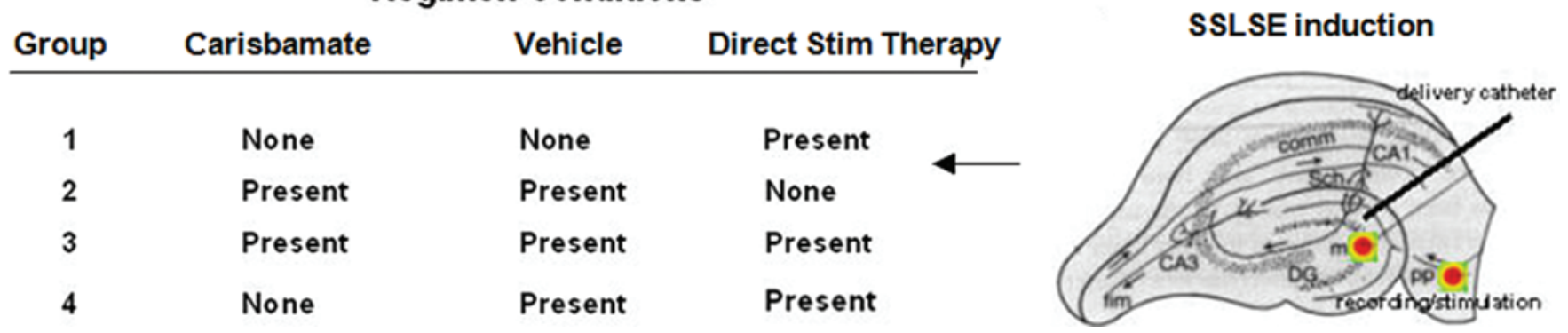

FIG. 3. Four treatment groups are outlined following induction of the SSLSE epilepsy model in the right PP-DG circuit. Stimulation (Stim) therapy was delivered in the absence (Groups 1 and 4) or presence (Groups 2 and 3) of on-demand 20-nl boluses of 100 $\mu \mathrm{g} / 50 \mu \mathrm{l}\left[{ }^{14} \mathrm{C}\right]$-carisbamate. Carisbamate was delivered at a rate of $500 \mathrm{nl} / \mathrm{min}$. The drug was always delivered in the right DG. No limit or cap was set for the maximum number of nano-boluses delivered. Figure is available in color online only. 
in M1-embedding matrix (Shandon, Inc.) while placed on dry ice. All frozen embedded brains were stored at $-70^{\circ} \mathrm{C}$. Ten-micrometer coronal whole brain slices containing the hippocampal formation and electrode tracts were sectioned using a Reichert-Jung 1800 cryostat (Leica, Inc.). The tissue sections from animals in Groups 2 and 3, which were exposed to $\left[{ }^{14} \mathrm{C}\right]$-carisbamate, were immediately mounted on twice-gelatinized glass slides. These slides were arranged on a flat surface and exposed to $\left[{ }^{14} \mathrm{C}\right]$-sensitive Hyperfilm (GE) in an x-ray film cassette at $4^{\circ} \mathrm{C}$ for 40 days. The film was then developed in a darkroom using D-19 developer (Kodak). Slides from Group 1 containing tissue sections obtained at the level of the dorsal hippocampal formation were placed on a blue LED transilluminator (BlueView, Vernier Software \& Technology) to better visualize the right hippocampal tract using the fluorescein-labeled chitosan nanoparticles injected prior to sacrifice.

\section{Statistical Analyses}

Statistical analyses (Prism v5.04, GraphPad Software) of the outcome measures were performed using nonparametric testing. A 2-way ANOVA was used to assess frequency and duration of spontaneous ADs occurring over 30 -minute epochs during the 10 -hour recording session for animals in each of the 4 condition groups.

\section{Results}

A mortality rate of $29 \%$ (8 of 28 animals implanted) was observed due to excessive electroclinical seizure ac- tivity either during the induction of SSLSE or near the onset of the therapy session. Therefore, these 8 animals were excluded from the analyses due to either nonexistent or virtually nonexistent recording sessions (less than several seconds of equivocal electrocerebral data). In addition, microelectrode failure in 4 animals resulted in no meaningful recordings following the induction of SSLSE. As a result, 16 animals were available for analysis and used in this study.

Two seizure patterns were observed following the completion of CHS. Specifically, animals exhibited either spontaneous electrographic seizures only or spontaneous electrographic seizures with a behavioral correlate (electroclinical seizures). Four animals demonstrated ictalassociated behaviors. Behavioral phenomena ranged from excessive grooming to repetitive rearing and falling similar to rodents with fully kindled (Stage 5) seizures. No behavioral differences were seen in the first half of the recording session compared with the final half of the session. Recurrent electrographic seizures for all 16 animals persisted throughout the recording session. SSLSE was considered to have been successfully achieved in these animals. Of note, the use of high-density contact microelectrodes allowed for an increased probability of sampling epileptiform propagation latencies from the hippocampal neuronal network both locally and contralaterally. The data in Fig. 4 demonstrate the termination of a seizure after evolving for several seconds throughout the right hippocampal formation subfields prior to propagating to

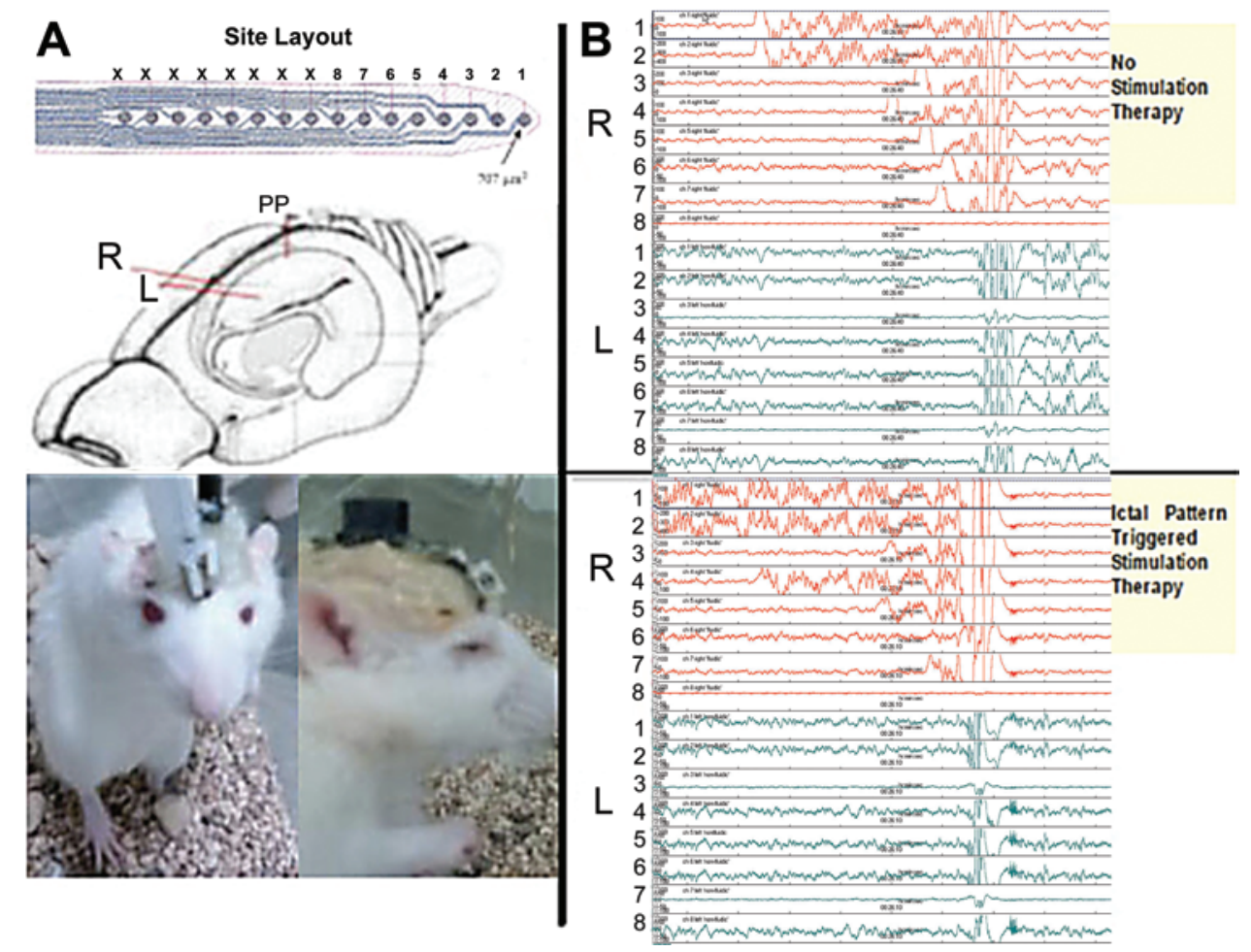

FIG. 4. The semichronic PP stimulating electrode was implanted $8 \mathrm{~mm}$ posterior to the right dorsal hippoocampal formation recording microelectrode array (A). A fixed stimulus frequency of $50 \mathrm{~Hz}$ with a pulse width of $1 \mathrm{msec}$ and burst duration of $100 \mathrm{msec}$ was maintained throughout all condition groups. Termination of a hippocampal seizure is demonstrated following delivery of direct stimulation current between $100-500 \mu \mathrm{A}$ (charge density of $1.0-4.5 \mu \mathrm{C} / \mathrm{cm}^{2}$ per phase) through the right PP bipolar electrode (B). Figure is available in color online only. 
the contralateral hippocampal formation. Therapy current (below the $\mathrm{AD}$ threshold with a calculated charge density of $1.0-4.5 \mu \mathrm{C} / \mathrm{cm}^{2}$ per phase) was delivered through the bipolar electrode implanted in the right PP. A fixed stimulus frequency of $50 \mathrm{~Hz}$, pulse width of $1 \mathrm{msec}$, and burst duration of $100 \mathrm{msec}$ were maintained throughout all condition groups.

Autoradiography demonstrated targeted ipsilateral hippocampal formation distribution of nano-bolused positive-pressure delivery of $\left[{ }^{14} \mathrm{C}\right]$-carisbamate concentrations from the distal shaft of the hybrid recording depth microelectrode shaft (Fig. 5). The cumulative volume of radiolabeled drug appeared to be consistently distributed within the confines of the crescent-shaped ipsilateral hippocampal formation without encroachment upon the ipsilateral thalamic or basal ganglia regions. Of note, autoradiography also demonstrated reflux of $\left[{ }^{14} \mathrm{C}\right]$-carisbamate along the shaft of the fluidic catheter. This reflux, or "back leak" was captured on the autoradiographs and extended caudad to the microcather insertion site. This appearance was likely caused by a pressure gradient coupled with decreased resistance along the shaft of the catheter. Such a finding is common with CED. ${ }^{40}$

The depth of the right hippocampal tract of the hybrid fluidic microelectrode shaft was confirmed by histology in Group 1 using fluorescein-labeled chitosan nanoparticles injected into the catheter immediately prior to sacrifice. Of note, a nano-bolused volume of the fluorescein-labeled

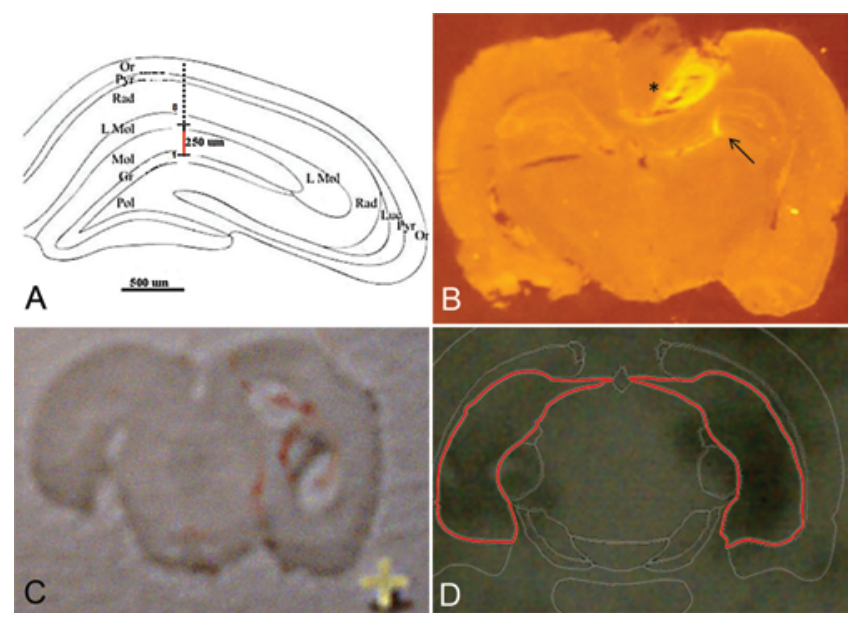

FIG. 5. Each animal was sacrificed and brain tissue was immediately frozen on dry ice in M1-embedding matrix after an 8- to 10-hour therapy session preceded by a 2-hour baseline recording period. For Group 1 animals, the tract in the right hippocampal formation (A) was better visualized using fluorescein-labeled chitosan nanoparticles injected immediately prior to sacrifice (arrow, B). Of note, reflux of the nanoparticles is shown along the hybrid microelectrode-catheter shaft as well as in the cortical mantle (asterisk, B). $\left[{ }^{14} \mathrm{C}\right]$-sensitive film was exposed to $10-\mu \mathrm{m}$ tissue sections on gelatinized glass microscope slides (C) for 40 days at $4^{\circ} \mathrm{C}$. The film was subsequently developed to demonstrate radiolabeled drug distribution as shown in $\mathbf{D}$ demarcated by a rat atlas coronal slice overlay at the corresponding level. Of note, reflux of $\left[{ }^{14} \mathrm{C}\right]-$ carisbamate occurred along the shaft of the fluidic catheter. This reflux, or "back leak" was captured on the autoradiographs and typically extended caudal to the microcatheter implant site (D). Figure is available in color online only. chitosan demonstrated reflux along the electrode shaft (Fig. 5B). In Groups 2-4, histology confirmed electrode placement in the left DG only. The left hippocampal tract (contralateral to drug delivery and PP stimulation) was more clearly demarcated in the $10-\mu \mathrm{m}$ frozen sections. This finding was due to an absence of potential mechanical deformation of tissue by repeated drug delivery, in contrast to the condition of the contralateral side. Mild mechanical deformation was consistently seen with the right electrode tract accommodating the hybrid recording microelectrode-catheter depth lead for Groups 2-4. This observation is important, since evoked potential-guided placement of the right DG hybrid microelectrode was useful due to dense ipsilateral PP white matter connectivity not seen contralaterally. Therefore, electrophysiological lead placement guidance was more heavily relied upon ipsilateral to PP stimulation. Of note, the dense commissural white matter tracts in rodents connecting the contralateral hippocampal formation is important for crosshemispheric communication, but is markedly less dense in primates. ${ }^{41}$

Delivery of on-demand focal stimulation therapy alone (Group 1) demonstrated marked variability in AD rate during the last $50 \%$ of the therapy session (Fig. 6). The animals in Group 4 (stimulation therapy + saline) appeared to demonstrate a decreased seizure frequency compared with Group 1 (stimulation therapy alone) throughout the entirety of the recording session. However, Group 4 did not demonstrate any change or trending from the beginning of the recording session until its completion. Whereas, the animals in Group 1 (stimulation therapy alone) demonstrated a downward trend, where the final 50\% of the recording session showed a significantly lower seizure frequency rate compared with the first $50 \%$ of the session $(\mathrm{p}<0.05)$ (Fig. 7). In contrast, delivery of nano-bolused carisbamate in the absence (Group 2) or presence (Group 3) of stimulation therapy revealed a low seizure frequency with minimal variability $(\mathrm{p}<0.05)$ compared with Groups 1 and 4 (Fig. 6, dashed red boxes). The duration of electrographic seizures demonstrated no consistent trend throughout the post-SSLSE recording-therapy sessions for all groups. In addition, seizure frequency and ictal duration were unrelated to the total number of therapy boluses delivered (the number of therapies/session ranged from 81 to 1832 for all groups, with a mean of 745).

To allow for adequate exposure of therapy to each treatment condition, differences in seizure frequency were assessed during the last $50 \%$ of the therapy session (21,600-36,000 seconds) over 30-minute (1800-second) epochs. A 2-way ANOVA on seizure frequency with treatment groups and time (final 50\% of therapy session) as the main factors showed a significant effect of groups $(\mathrm{F}(3,12)$ $=10.53, \mathrm{p}=0.001)$ and time $(\mathrm{F}(8,96)=3.366, \mathrm{p}=0.002)$, as well as a significant interaction between them $(\mathrm{F}(24,96)$ $=2.181, \mathrm{p}=0.004)$. Multiple comparisons indicated that during pulsatile delivery of carisbamate in tandem with closed-loop direct stimulation therapy to the right PP, the mean group seizure frequency significantly decreased compared with closed-loop stimulation therapy alone ( $p$ $<0.001)$. In addition, seizure frequency decreased in the carisbamate group $(\mathrm{p}<0.05)$ compared with stimulation 

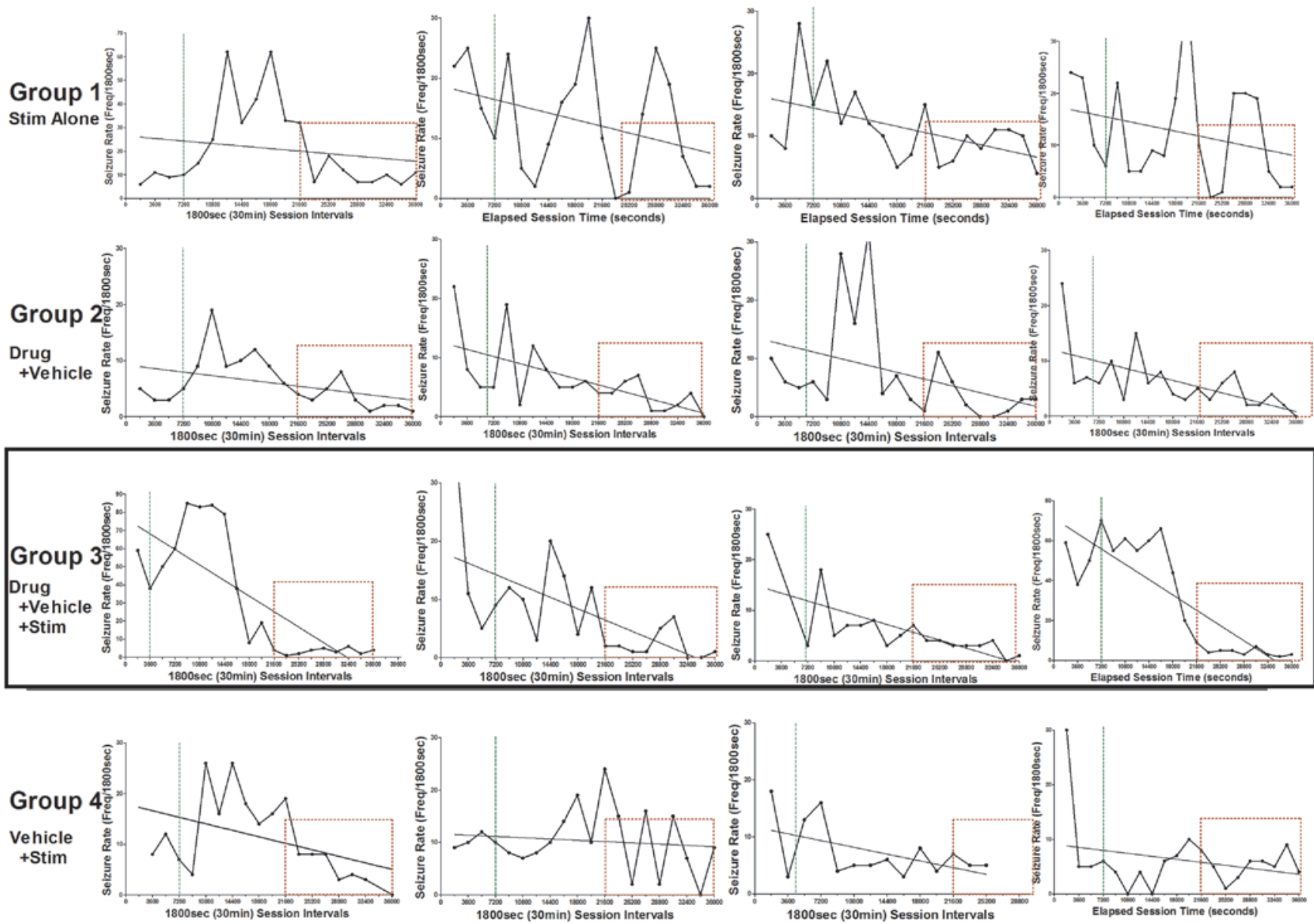

FIG. 6. Electrographic seizure frequency per 30-minute interval was assessed over an 8- to 10-hour therapy session, following a 2-hour (7200-second) post-SSLSE/pretherapy baseline interval (green dashed line). Red boxes indicate the last $50 \%$ of the therapy session, which was used for comparisons of seizure frequency between animals and groups. The entirety of each ECOG session with video was reviewed by a blinded experienced electroencephalographer (M.A.R.). Figure is available in color online only.

therapy alone (Fig. 7). No relationship of treatment group with duration of electrographic seizures or severity of behavioral seizures was observed.

\section{Discussion}

To the best of our knowledge, this study is the first to evaluate the effects of combining direct responsive neurostimulation therapy and on-demand intracerebral CED in a self-sustained focal-onset epilepsy model in rodents. Equally important, this study demonstrated the feasibility of delivering direct neurostimulation therapy to the ipsilateral PP to abort the electrocerebral ictal onset detected at a distance via communicating white matter pathways in both the ipsi- and contralateral DG. The animal model employed takes into account a white matter-connected bihemispheric epileptic circuit, and not simply the presumed gray matter "focus." 22 Unlike open-loop, or scheduled neurostimulation therapy, closed-loop stimulation limits the stimulus to the immediate pre-ictal or early ictal period. ${ }^{39}$ Such microsecond bursts of therapeutic stimulation result in an overall reduction in cumulative current delivered over a prolonged interval. In effect, exposure to prolonged epochs of stimulation is minimized.9,32 Finally, this study was limited to the acute effects of responsive local stimulation therapy. Proposed mechanisms explaining acute-onset efficacy include conduction blockade, synaptic inhibition, synaptic depression, and overriding pathophysiological neural network activity. ${ }^{35}$

In tandem with responsive direct neurostimulation therapy, this study demonstrated augmented efficacy with ondemand pulsatile intracerebral delivery of carisbamate in the ipsilateral epileptogenic DG. In particular, on-demand delivery of carisbamate to a critical location in the seizure circuit was shown to impact the neurophysiology of the seizure-prone network. The combination of closed-loop neurostimulation therapy and on-demand nano-volume bolused intracerebral carisbamate therapy (Group 3) demonstrated a statistically significant decrease in electrocerebral seizure frequency as compared with closed-loop stimulation therapy alone (Group 1). The isolation of $\left[{ }^{14} \mathrm{C}\right]-$ carisbamate distribution to the hippocampal formation underscores the ability of targeted nano-bolused drug delivery to stabilize an epileptic circuit while sparing exposure of nonepileptic brain regions to drug. In addition, the com- 


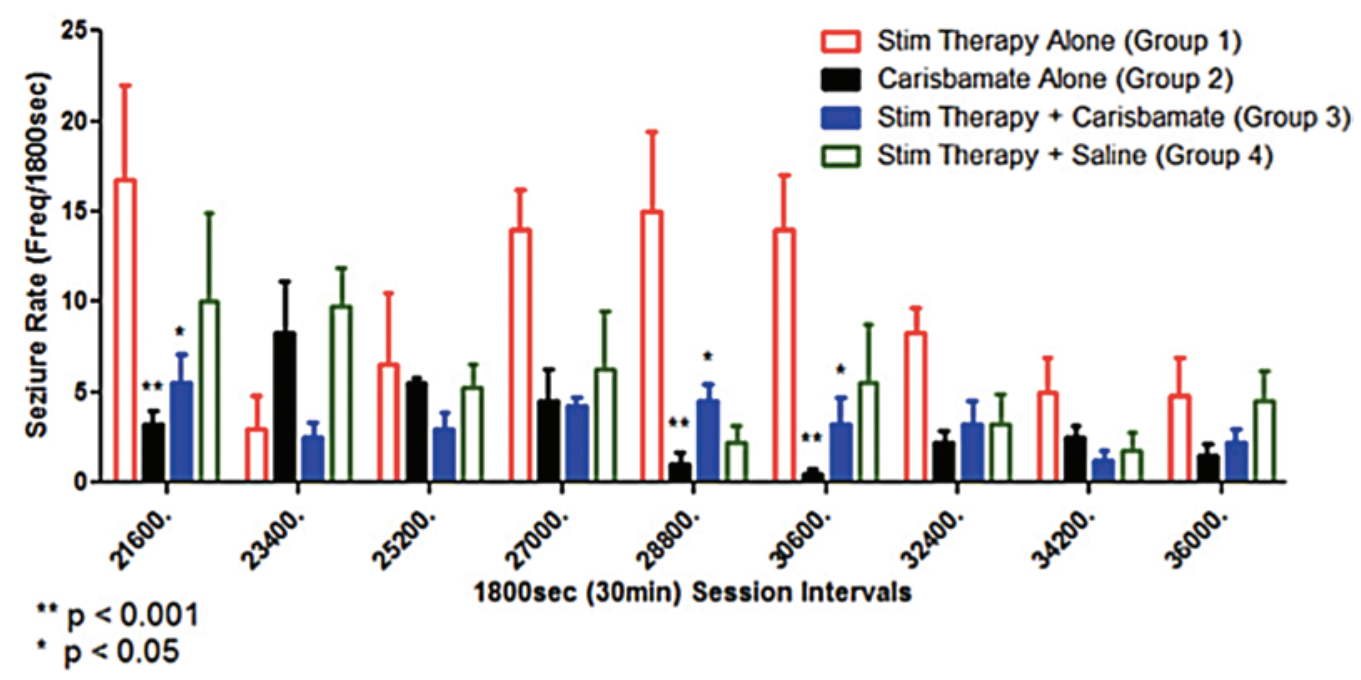

FIG. 7. Significant differences in seizure frequency were seen with carisbamate delivery alone (carisbamate-only, Group 2) and stimulation therapy in tandem with carisbamate delivery (Group 3) in comparison to stimulation therapy alone (Group 1). Figure is available in color online only.

bination of responsive direct neurostimulation therapy and on-demand intracerebral drug delivery potentially reduces desensitization and the volume of AED introduced outside of the intended target.

In previous studies involving animal epilepsy models, AEDs were administered using CED strategies in either a single dose or in a chronic fashion over a period of days to weeks. Van Dycke et al. ${ }^{39}$ demonstrated that, in rats kindled through the intraperitoneal kainic acid status epilepticus model, chronic direct hippocampal delivery of adenosine reduced the mean daily seizure frequency by $33 \%$. In a rat amygdala-kindling model, Gasior et al. ${ }^{6}$ demonstrated that localized CED infusion of $\omega$-conotoxin GVIA and $\omega$-conotoxin MVIIA into the amygdala produced a long-lasting elevation in the AD threshold of fully kindled rats. The toxins caused a dose-dependent reduction in seizure parameters, including AD duration and duration of behavioral limbic seizure activity. Furthermore, Rogawski et al. ${ }^{33}$ reported that, in a fully amygdala-kindled rat, CED of botulinum neurotoxins $\mathrm{A}$ and $\mathrm{B}$ into the amygdala resulted in significant, dose-dependent elevation in the $\mathrm{AD}$ threshold and decreases in AD duration that lasted from 3 to 35 days postinfusion.

The primary advantage of CED over diffusion-driven mechanisms is that therapeutic agents can be transported more rapidly through the porous extracellular space. Despite the increased transport rate, minimal tissue damage has been observed on histological analysis. Mechanical deformation of brain adjacent to the drug-delivery microcatheter seen in our study may be due to not limiting pulsatile drug delivery to a maximum number of nanoboluses over a given time interval (Fig. 5A). However, as in our study, no significant neurological deficits are typically described after CED in rodent and nonhuman primate models. ${ }^{10,12,16,21,28,40}$

From a microfluidic mechanistic perspective, a steep drop-off in CED has been observed at the distribution boundaries; $8,17,36,37$ however, it is important to note that a concentration gradient centered at the catheter tip is main- tained. ${ }^{37}$ While it has been previously reported that solutes are distributed homogeneously throughout the infusion volume regardless of the solute's molecular weight, $, 4,8,28$ the governing transport equation describing this phenomenon must be considered, as seen in the convection-diffusion equation (Equation 1) below.

$$
\partial \mathrm{c} / \partial \mathrm{t}+\overrightarrow{\mathrm{V}} \cdot \nabla \mathrm{c}=\nabla \cdot(\mathrm{D} \nabla \mathrm{c})
$$

Eq. 1

In this equation, $\mathrm{c}=$ variable of interest (drug concentration), $\mathrm{t}=$ time, $\mathrm{D}=$ diffusivity (also called diffusion coefficient), $\vec{V}=$ average velocity of the fluid flow, $\nabla$ represents gradient, and $\nabla \cdot$ represents divergence.

In CED, both convection and diffusion govern the transport of a solute within the bulk flow. While convection's influence $(\overrightarrow{\mathrm{V}} \cdot \nabla \mathrm{c})$ on solute transport, caused by the velocity vector $(\overrightarrow{\mathrm{V}})$ of the bulk flow, is initially significantly larger on a global scale, the diffusion term $(\partial \mathrm{c} / \partial \mathrm{t})$ cannot be neglected. As the infusate's volume and distribution increase, convection's role in the transport of the drug diminishes. ${ }^{11}$ In light of this, these governing forces must be balanced to successfully deliver therapeutic agents to a defined area via CED. Successfully balancing these forces can potentially deliver therapy to specific regions within the CNS with minimal injury, while limiting both exposure of nontargeted regions and systemic toxicity.

\section{Conclusions}

This study's dual treatment strategy of responsive direct electrical stimulation coupled with pulsatile drug delivery yields many advantages. It decreases spontaneous expression of seizure activity, eliminates dose-limiting adverse side effects experienced due to systemic delivery of anticonvulsants, and minimizes desensitization and loss of AED binding sites within both neurons and glial cells in the absence of persistent saturating drug.

This work demonstrated the feasibility of stabilizing an active bihemispheric limbic epileptic circuit by combining 
2 separate seizure-suppression therapies - namely, closedloop responsive direct neurostimulation and on-demand CED of targeted carisbamate. Closed-loop direct stimulation of unilateral afferent hippocampal white matter pathways concurrent with on-demand intracerebral delivery of nano-bolused carisbamate can significantly decrease electrographic seizure frequency in an active epileptogenic network extending to the contralateral hemisphere. Additionally, the introduction of intracerebral pulsatile carisbamate delivery can stabilize seizure frequency variability compared with closed-loop direct stimulation therapy alone.

Future directions for overcoming limitations of CED include potentially steering nanocarrier-based therapeutic molecules through dense pathological tissue employing electrical or magnetic fields. Such electromagnetic fields may themselves demonstrate an additive or synergistic effect on quieting potentially extensive epileptogenic neural circuits. Future studies will need to better delineate the underlying mechanisms of neurostimulation therapy to develop the tools for determining optimal stimulation parameters. ${ }^{42}$ In addition, timing of delivery, relative to the early electrocerebral ictal signature or pre-ictal period, must be optimized. . $^{15,23,24}$ As the molecular underpinnings of neuromodulation evolve, and innovative technologies are realized, the most effective therapeutic system will not be based on a single method. Instead, it is likely that a combination of technologies will be integrated which can be optimized with individual specificity.

\section{References}

1. Amaral DG, Witter MP: The three-dimensional organization of the hippocampal formation: a review of anatomical data. Neuroscience 31:571-591, 1989

2. Andermann E, Andermann F, Meyvisch P, Vandendriessche A, Schiemann Delgado J: Antiepileptic drugs: all ages. Epilepsia 46 (Suppl s8):205-222, 2005

3. Bobo RH, Laske DW, Akbasak A, Morrison PF, Dedrick RL, Oldfield EH: Convection-enhanced delivery of macromolecules in the brain. Proc Natl Acad Sci U S A 91:2076-2080, 1994

4. Chen MY, Lonser RR, Morrison PF, Governale LS, Oldfield EH: Variables affecting convection-enhanced delivery to the striatum: a systematic examination of rate of infusion, cannula size, infusate concentration, and tissue-cannula sealing time. J Neurosurg 90:315-320, 1999

5. Devinsky O: Patients with refractory seizures. N Engl J Med 340:1565-1570, 1999

6. Gasior M, White NA, Rogawski MA: Prolonged attenuation of amygdala-kindled seizure measures in rats by convectionenhanced delivery of the $\mathrm{N}$-type calcium channel antagonists omega-conotoxin GVIA and omega-conotoxin MVIIA. J Pharmacol Exp Ther 323:458-468, 2007

7. Grabenstatter HL, Ferraro DJ, Williams PA, Chapman PL, Dudek FE: Use of chronic epilepsy models in antiepileptic drug discovery: the effect of topiramate on spontaneous motor seizures in rats with kainate-induced epilepsy. Epilepsia 46:8-14, 2005

8. Groothuis DR, Ward S, Itskovich AC, Dobrescu C, Allen $\mathrm{CV}$, Dills C, et al: Comparison of ${ }^{14} \mathrm{C}$-sucrose delivery to the brain by intravenous, intraventricular, and convectionenhanced intracerebral infusion. J Neurosurg 90:321-331, 1999

9. Hasulak N, Breeden M, Nathwani N, Wang C, Saghyan A,
Skarpaas T, et al: Continuous video/EEG system with closed loop stimulation for rat models of epilepsy. Presented at the 65th annual meeting American Epilepsy Society, Baltimore, MD, 2011 (Abstract) (https://www.aesnet.org/meetings_ events/annual_meeting_abstracts/view/15096) [Accessed January 12,2015 ]

10. Inoue T, Yamashita Y, Nishihara M, Sugiyama S, Sonoda Y, Kumabe T, et al: Therapeutic efficacy of a polymeric micellar doxorubicin infused by convection-enhanced delivery against intracranial 9L brain tumor models. Neuro Oncol 11:151-157, 2009

11. Ivanchenko O, Sindhwani N, Linninger AA: Exact solution of the diffusion-convection equation in cylindrical geometry. AIChE J 58:1299-1302, 2012

12. Krauze MT, Mcknight TR, Yamashita Y, Bringas J, Noble CO, Saito R, et al: Real-time visualization and characterization of liposomal delivery into the monkey brain by magnetic resonance imaging. Brain Res Brain Res Protoc 16:20-26, 2005

13. Kwan P, Brodie MJ: Early identification of refractory epilepsy. N Engl J Med 342:314-319, 2000

14. Levy RH, Mattson RH, Meldrum BS, Perucca E (eds): Antiepileptic Drugs, ed 5. Philadelphia: Lippincott Williams \& Wilkins, 2002

15. Litt B, Echauz J: Prediction of epileptic seizures. Lancet Neurol 1:22-30, 2002

16. Lonser RR, Corthésy ME, Morrison PF, Gogate N, Oldfield EH: Convection-enhanced selective excitotoxic ablation of the neurons of the globus pallidus internus for treatment of parkinsonism in nonhuman primates. J Neurosurg 91:294302, 1999

17. Lonser RR, Walbridge S, Garmestani K, Butman JA, Walters HA, Vortmeyer AO, et al: Successful and safe perfusion of the primate brainstem: in vivo magnetic resonance imaging of macromolecular distribution during infusion. J Neurosurg 97:905-913, 2002

18. Löscher W, Schmidt D: Modern antiepileptic drug development has failed to deliver: ways out of the current dilemma. Epilepsia 52:657-678, 2011

19. Lothman EW, Bertram EH, Bekenstein JW, Perlin JB: Selfsustaining limbic status epilepticus induced by 'continuous' hippocampal stimulation: electrographic and behavioral characteristics. Epilepsy Res 3:107-119, 1989

20. Mangubat E, Rossi M: On-demand pulsatile intracerebral delivery of carisbamate concurrent with closed-loop direct neurostimulation therapy in a self-sustained limbic status epilepticus (SSLSE) rat model. Presented at the 64th annual meeting of the American Epilepsy Society, San Antonio, TX, 2010 (Abstract) (https://www.aesnet.org/meetings_ events/annual_meeting_abstracts/view/13076) [Accessed January 12,2015$]$

21. Mardor Y, Last D, Daniels D, Shneor R, Maier SE, Nass D, et al: Convection-enhanced drug delivery of interleukin-4 Pseudomonas exotoxin (PRX321): increased distribution and magnetic resonance monitoring. J Pharmacol Exp Ther 330:520-525, 2009

22. Mazarati AM, Wasterlain CG, Sankar R, Shin D: Self-sustaining status epilepticus after brief electrical stimulation of the perforant path. Brain Res 801:251-253, 1998

23. Mormann F, Andrzejak RG, Elger CE, Lehnertz K: Seizure prediction: the long and winding road. Brain 130:314-333, 2007

24. Morrell M: Brain stimulation for epilepsy: can scheduled or responsive neurostimulation stop seizures? Curr Opin Neurol 19:164-168, 2006

25. Morrell MJ: Responsive cortical stimulation for the treatment of medically intractable partial epilepsy. Neurology 77:1295-1304, 2011

26. Morrison PF, Laske DW, Bobo H, Oldfield EH, Dedrick RL: 
High-flow microinfusion: tissue penetration and pharmacodynamics. Am J Physiol 266:R292-R305, 1994

27. Nilsen KE, Cock HR: Focal treatment for refractory epilepsy: hope for the future? Brain Res Brain Res Rev 44:141-153, 2004

28. Occhiogrosso G, Edgar MA, Sandberg DI, Souweidane MM: Prolonged convection-enhanced delivery into the rat brainstem. Neurosurgery 52:388-394, 2003

29. Ono T, Moshé SL, Galanopoulou AS: Carisbamate acutely suppresses spasms in a rat model of symptomatic infantile spasms. Epilepsia 52:1678-1684, 2011

30. Paxinos G, Watson C: The Rat Brain in Stereotaxic Coordinates, ed 4. London: Academic Press, 1998

31. Racine RJ: Modification of seizure activity by electrical stimulation. II. Motor seizure. Electroencephalogr Clin Neurophysiol 32:281-294, 1972

32. Raghunathan S, Gupta SK, Ward MP, Worth RM, Roy K, Irazoqui PP: The design and hardware implementation of a low-power real-time seizure detection algorithm. J Neural Eng 6:056005, 2009

33. Rogawski MA: Convection-enhanced delivery in the treatment of epilepsy. Neurotherapeutics 6:344-351, 2009

34. Rossi MA: Targeting anti-epileptic drug therapy without collateral damage: nanocarrier-based drug delivery. Epilepsy Curr 12:199-200, 2012

35. Rossi MA, Stebbins G, Murphy C, Greene D, Brinker S, Sarcu D, et al: Predicting white matter targets for direct neurostimulation therapy. Epilepsy Res 91:176-186, 2010

36. Saito R, Krauze MT, Noble CO, Tamas M, Drummond DC, Kirpotin DB, et al: Tissue affinity of the infusate affects the distribution volume during convection-enhanced delivery into rodent brains: implications for local drug delivery. $\mathbf{J}$ Neurosci Methods 154:225-232, 2006

37. Sindhwani N, Ivanchenko O, Lueshen E, Prem K, Linninger AA: Methods for determining agent concentration profiles in agarose gel during convection-enhanced delivery. IEEE Trans Biomed Eng 58:626-632, 2011
38. Srivastava AK, White HS: Carbamazepine, but not valproate, displays pharmacoresistance in lamotrigine-resistant amygdala kindled rats. Epilepsy Res 104:26-34, 2013

39. Van Dycke A, Raedt R, Dauwe I, Sante T, Wyckhuys T, Meurs A, et al: Continuous local intrahippocampal delivery of adenosine reduces seizure frequency in rats with spontaneous seizures. Epilepsia 51:1721-1728, 2010

40. White E, Bienemann A, Malone J, Megraw L, Bunnun C, Wyatt M, et al: An evaluation of the relationships between catheter design and tissue mechanics in achieving high-flow convection-enhanced delivery. J Neurosci Methods 199:8797,2011

41. White G, Levy WB, Steward O: Evidence that associative interactions between synapses during the induction of longterm potentiation occur within local dendritic domains. Proc Natl Acad Sci U S A 85:2368-2372, 1988

42. Wyckhuys T, Boon P, Raedt R, Van Nieuwenhuyse B, Vonck K, Wadman W: Suppression of hippocampal epileptic seizures in the kainate rat by Poisson distributed stimulation. Epilepsia 51:2297-2304, 2010

\section{Author Contributions}

Conception and design: Rossi, Mangubat. Acquisition of data: Rossi, Mangubat, Kellogg. Analysis and interpretation of data: all authors. Drafting the article: all authors. Critically revising the article: Rossi, Mangubat, Harris. Reviewed submitted version of manuscript: Rossi, Mangubat, Harris. Approved the final version of the manuscript on behalf of all authors: Rossi. Statistical analysis: Rossi, Mangubat. Study supervision: Rossi.

\section{Correspondence}

Marvin A. Rossi, 1725 W. Harrison St., Ste. 885, Chicago, IL 60612.email: marossi@usa.net. 